\title{
Is Reasonable of the Criteria for the Release Isolation and Discharge of the Latest Clinical Practice Guidelines of 2019-nCov in China?
}

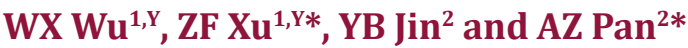 \\ ${ }^{1}$ Department of Radiology, The First People's Hospital of Foshan, China \\ ${ }^{2}$ Department of Clinical research institute, The First People's Hospital of Foshan, China \\ ${ }^{Y}$ These authors contributed equally as first authors
}

*Corresponding author: ZF Xu, Department of Radiology, The First People's Hospital of Foshan, Guagndong, 528000, China

AZ Pan, Department of Radiology, The First People's Hospital of Foshan, Guagndong, 528000, China

\begin{abstract}
ARTICLE INFO
Received: webruary 26, 2020

Published: March 05, 2020

Citation: WX Wu, ZF Xu, YYB Jin, AZ Pan. Is Reasonable of the Criteria for the Release Isolation and Discharge of the Latest Clinical Practice Guidelines of 2019-nCov in China?. Biomed J Sci \& Tech Res 26(2)-2020. BJSTR. MS.ID.004336.
\end{abstract}

Keywords: 2019-nCoV; Pneumonia; Guideline; Isolation; Chest CT

\section{ABSTRACT}

Objective:2019-nCoV cause respiratory disease and severe pneumonia and is characterized by long incubation period, diversified symptoms, high false negative rate of nucleic acid, strong infectivity and diversified transmission routes. Therefore, we focused on the scientificity and reasonability of the criterias for the release isolation and discharge of patients in guideline published by National Health Commission of China.

Methods: 6 patients with suspected or confirmed New Coronavirus Pneumonia (NCP) were collected, and were divided into two group (Cured group and Isolation group).Clinical manifestations(such as fever, cough, anhelation, etc.), nucleic acid test and chest CT findings of all patients in two subgroups were analyzed and compared.

Results: The study found that the patients with 2019-nCoV met the standards of release isolation and discharge in guidelines. However, their clinical manifestations, nucleic acid tests and chest CT findings all overlapped with those of suspected or confirmed cases $(\mathrm{p}=0.075)$. Especially, the nucleic acid tests and chest CT findings, the most important criteria for early diagnosis and explicit diagnosis of NCP with a high degree of overlap.

Conclusion: It is necessary to face up to these cured patients, whether they would bring the hidden danger of infection. Standardized centralized isolation and management (7-14 days) for them should be continued, and chest CT and nuclide testing should be conducted again.

\section{Introduction}

In December 2019, unexplained pneumonia occurred in Wuhan, HuBei, China, and most of them had direct exposure to Huahai Seafood Market, and with family aggregation, human-to-human transmission and strong infectivity [1-3]. As of by 20 January 2020, the new virus was named 2019 Novel Coronavirus (2019-nCov) by the world health organization (WHO). The number of people diagnosed with 2019-ncov infections has exploded in the past month, making it a public health event threatening the world. As of 16 February 2020, the novel coronavirus (2019-nCoV) pneumonia has caused 79675 confirmed cases including 1666 deaths and 9425 cured cases, and 8228 suspected cases, in mainland China. The number of cures is still relatively low compared to the number of confirmed cases, and treatment is still a long way off. Pneumonia protocols for the new coronavirus infection are also being updated with the change of the epidemic situation and the deepening of the understanding of new coronavirus pneumonia (NCP).According to the diagnostic criteria of the latest diagnosis and treatment guidelines (the $5^{\text {th }}$ Edition) in China [4].

Two different criteria were advanced for NCP patients in or out Hubei province. For cases in Hubei province, once positive 
nucleic acid was found in nasopharyngeal swabs, sputum, lower respiratory tract secretions, blood, feces and other specimens, NCP was confirmed immediately. Moreover, chest CT in the diagnosis of NCP was clarified and taken very seriously, because of very low sensitivity of nucleic acid detection (30-50\%) resulted in many clinical patients missed diagnosis. Therefore, even the nucleic acid of 2019-nCov is negative, once had typical epidemiological history and typical pulmonary CT manifestations, the patient must be considered as clinical cases. However, for cases outside Hubei province, the twice negative new coronavirus nucleic acid can be contact isolation, but for patients with lung lesions contact isolation will pose a great potential risk to the public. In addition, in the fifth edition of the diagnosis and treatment guideline [4], the isolation can be lifted if the NCP patients meet with the isolation and discharge criteria.

At present, to our knowledge, no study about chest CT findings of patients after release and discharge have been reported, meanwhile, the reasonable of fifth edition of guidelines for the release isolation and discharge has not been evaluated, and there is still a lack of standardized management and follow-up systems for cured patients. In this study, cases data of patients discharged from isolation, suspected and confirmed patients were collected, and clinical data and CT image data of the two groups of patients were analyzed and compared, and to discuss the safety and scientificity of the criteria for the release isolation and discharge of the latest clinical practice guidelines of 2019-nCov in China.

\section{Materials and Methods}

\section{Patients}

Our institutional review board waived written consent for this retrospective case series involved no potential risk to patients. To avert any potential breach of confidentiality, no link between the patients and the researchers was made available. Clinical data and imaging data of 6 patients with suspected or confirmed NCP were collected. The 6 patients were divided into cured group (discharge group) and isolation group (isolation inpatient and non-discharge group). The details about diagnosis and treatment of these patients in the two groups were as follows:

\section{Cured Group ( 3 cases)}

a. Case 1: Man, 40Y, was admitted to the hospital with fever and cough for 10 days. The patient's wife (Case 2) developed fever on the way back from Wuhan. After admission, the new coronavirus nucleic acid tested positive. Multiple CT scans were performed (Figure 1) and the pulmonary lesions were significantly absorbed, thrice nucleic acid tests for new coronavirus infection were negative. The patient was released from isolation and discharged without fever and occasional cough.

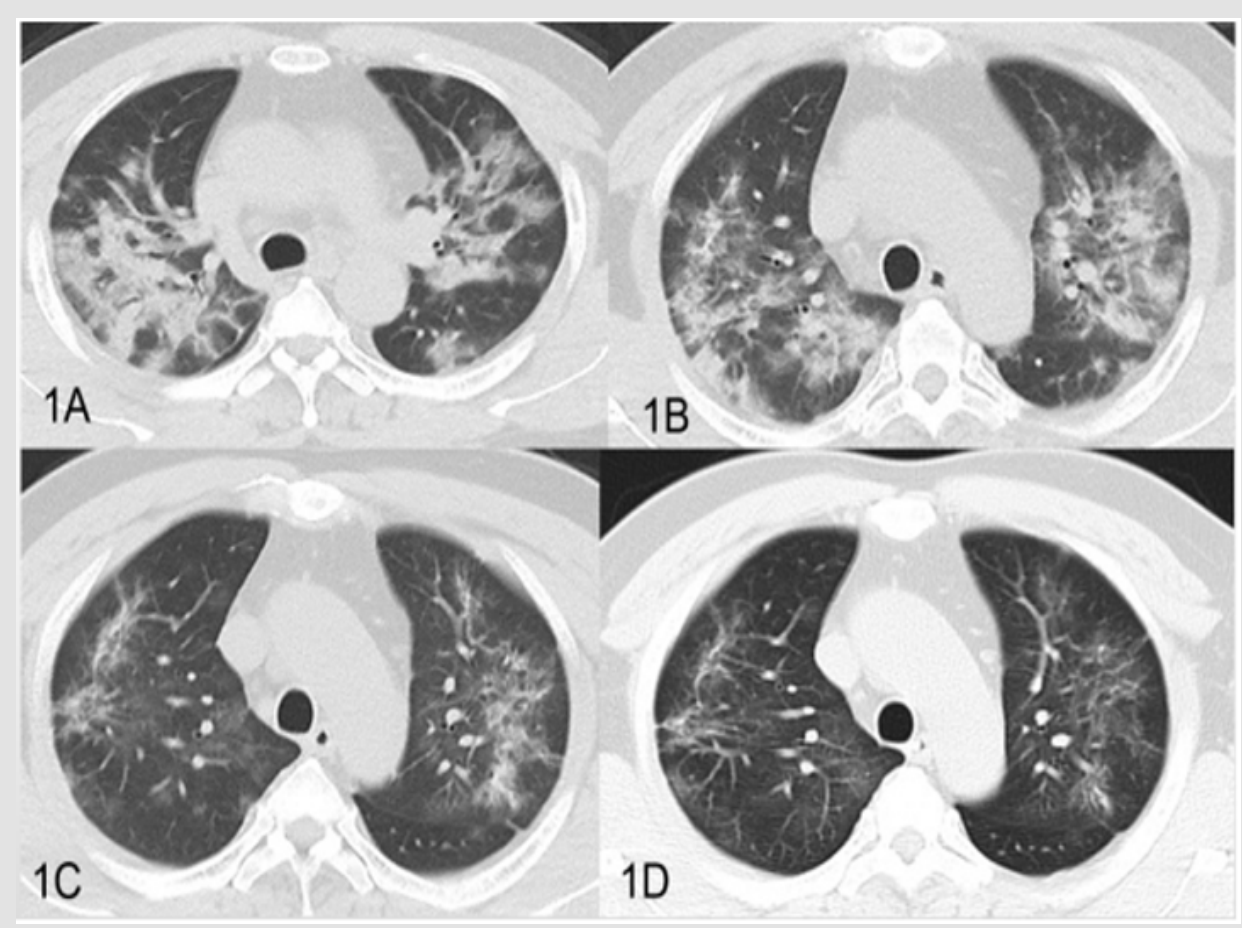

Figure 1: CT images of cured group case1, 40-year old male.

A. Patchy, ground hyaline lesions in both lungs, partial consolidation (2020/1/20).

B. Bilateral lung plaques and ground glass gradually reduced absorption $(2020 / 1 / 25)$.

C. Double pulmonary disease with obvious absorption, progressive fibrosis (2020/2/1).

D. Multiple ground glass slightly absorbed in both lungs, residual ground glass and fiber strips (2020/2/5). 
b. Case 2: Woman, 40Y, was admitted to the hospital with fever and cough for 2 weeks. The patient was twice negative for the new coronavirus nucleic acid (more than 24 hours apart). The patient was released from isolation and discharged with a normal temperature for 3 days, no cough, chest tightness and other symptoms.

c. Case 3: Woman 26Y, with a cough for 3 days, returned from Wuhan and her husband was diagnosed with NCP. Seven respiratory viruses including influenza $\mathrm{A}$ and $\mathrm{B}$ were negative, and thrice nucleic acid tests for new coronavirus infection were negative. Plain chest CT scans indicated pathological absorption. The patient was released from isolation and discharged without fever and occasional cough.

\section{Isolation Group ( 3 cases)}

a. Case1 Man, 56Y, with fever, dry cough for 8 days, exposed to Wuhan, was admitted to the hospital and new coronavirus nucleic acid tested positive in other hospital. But twice new coronavirus nucleic acid tests were negative duration of hospital stay. The patient underwent plain chest CT scan (Figure 2). The patient remains in isolation.
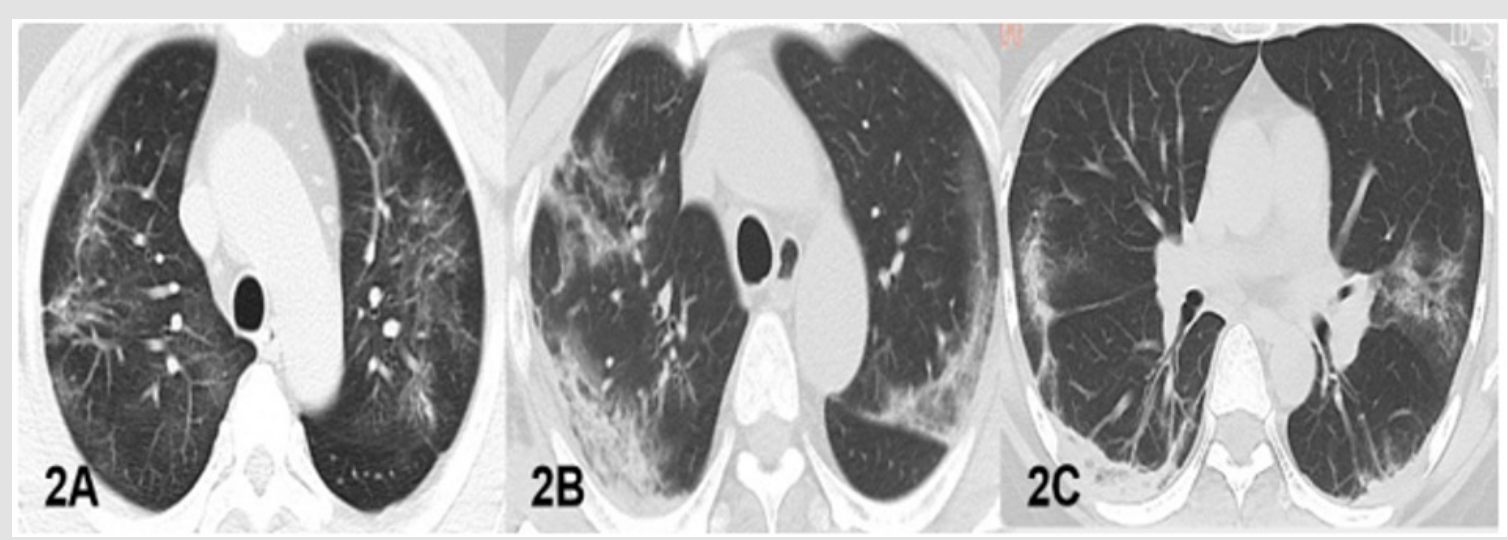

Figure 2: CT findings of NCP.

A. Case1 in cured group, chest CT displayed multiple fiber strip and ground glass shadow in both lungs.

B. B-C. Case1 and 3 in isolation group and, the chest CT displayed multiple patchy, ground-glass densified shadows with partial consolidation, a little fiber under the pleura in both lungs.

b. Case 2 Woman 56Y, with fever and dry cough for 1 day. The husband had contact with people from wuhan. She underwent three plain chest CT scans (Figure 3), with slight absorption of pulmonary lesions. Both swabs and deep sputum samples and blood samples were negative for the new coronavirus nucleic acid. The patients were still hospitalized for observation.

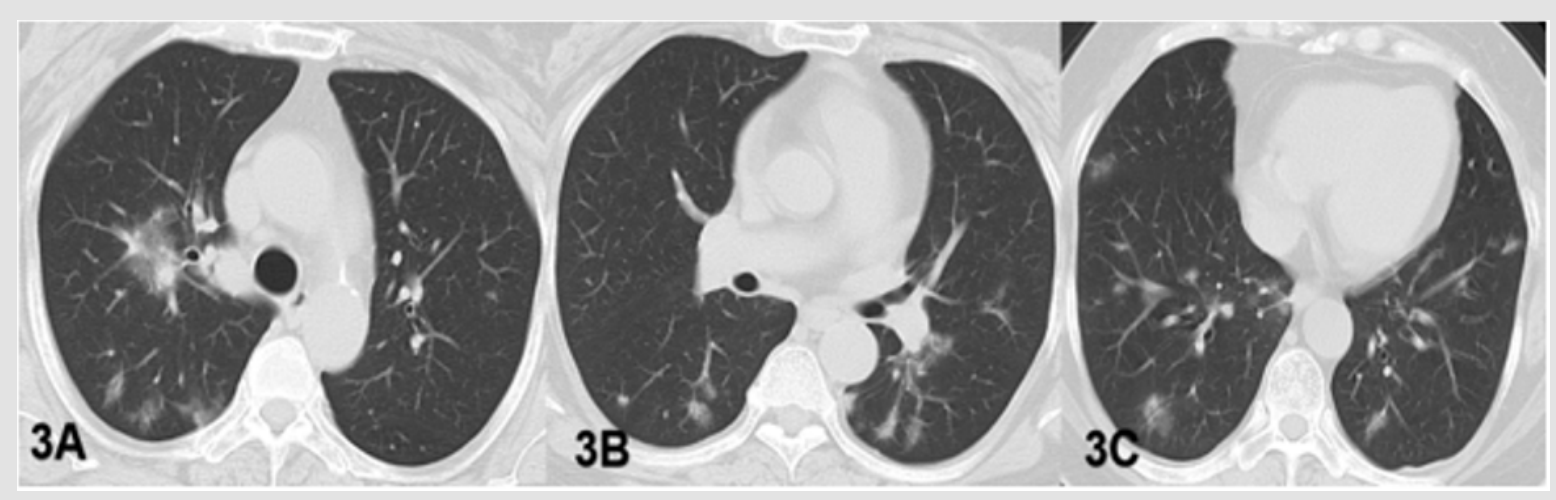

Figure 3: CT findings of case 2 in isolation group. 56-year old woman whose husband had contact with a Wuhan resident. Her CT scan showed normal at the beginning of the disease $(2020 / 1 / 25)$.

(A-C): 7 days later, chest CT images of this patient displayed multiple patchy and nodular ground glass shadows in both lungs (2020/2/1).

c. $\quad$ Case 3 Woman 38Y, with fever, dry cough for 13 days and shortness of breath for 7 days, returned from Hubei province. The new coronavirus nucleic acid was positive in other hospital.
But three times of new coronavirus nucleic acid tests were all negative. The patient underwent chest CT (Figure 2). The patient remains in isolation. 


\section{Chest CT Examination and CT Review}

All the patients had received CT examination when they were hospitalized, and the CT scanning model of all the patients was Toshiba Medical Systems(Tokyo, Japan).The scanning layer thickness $1 \mathrm{~mm}$, layer space $0 \mathrm{~mm}$, matrix $512 \times 512$, FOV $41.20 \mathrm{~mm}$, and with $120 \mathrm{KV}$ and $578 \mathrm{mAs}$. All CT images were reviewed by two radiologists with a 5 years history of cardiothoracic diagnosis. If the results of two radiologists are inconsistent, the decision should be made by a third cardiologist with more than 10 years of experience.

\section{Data Processing and Statistical Analysis}

Clinical manifestations such as fever, cough, anhelation and nucleic acid, and also CT findings such as GGO, consolidation and fibrosis, were all adopt the scoring method for the evaluation. The negative record was 0 score and the positive record was 1 score. In addition, number of lobes affected as the score for evaluation. All statistical analyses were performed by using the Statistical Package for Social Sciences, Version 17.0 (SPSS 17.0). The patients' scores in two group were compared based on the Mann-Whitney $U$ test. $\mathrm{P}<0.05$ was considered statistically significant.

\section{Results}

\section{Clinical Manifestations}

All patients in the cured group had no fever for more than 3 days when they were discharged from the hospital, whereas the patients in the isolation treatment group still had a fever. Of 3 patients in cured group, 2 cases (2/3) still underwent dry cough, and all patients presented cough in isolation treatment group. Besides, 3 patients in the cured group showed no significant shortness of breath, while 2 patients in the isolation group showed significant shortness of breath. Compared with the inpatients, typical clinical manifestations such as fever, cough and anhelation were significantly reduced or disappeared for patients in cured group, met with the standards for release isolation and discharge. All the patients in the cured group, twice nucleic acid detection was both negative. Whereas, this study displayed patients who need isolation and treatment were still negative for two- or three-times nucleic acid detection. These results showed that nucleic acid as the gold standard for 2019-NCP diagnosis can be negative in infectious patients need to be isolated (Table 1).

Table 1: Comparison and scores of clinical manifestations and chest CT features between cured group and isolation group.

\begin{tabular}{|c|c|c|c|c|c|c|c|c|}
\hline \multirow[t]{2}{*}{$\begin{array}{l}\text { Patients' } \\
\text { Data }\end{array}$} & \multicolumn{4}{|c|}{ Cured Group (cases) } & \multicolumn{4}{|c|}{ Isolation Group(cases) } \\
\hline & 1 & 2 & 3 & & 1 & 2 & 3 & $P$ value \\
\hline Age & 40 & 40 & 26 & & 56 & 56 & 38 & \\
\hline Sex & M & $\mathrm{F}$ & $\mathrm{F}$ & & M & $\mathrm{F}$ & $\mathrm{F}$ & \\
\hline \multicolumn{9}{|l|}{ Cincal data } \\
\hline Fever & 0 & 0 & 0 & & 1 & 1 & 1 & \\
\hline Cough & 1 & 0 & 1 & & 1 & 1 & 1 & \\
\hline \multicolumn{9}{|l|}{ Anhelation } \\
\hline 0 & 0 & 0 & & 1 & 0 & 1 & & \\
\hline Nucleic acid & 0 & 0 & 0 & & 0 & 0 & 0 & \\
\hline \multicolumn{9}{|l|}{ CT finding } \\
\hline GGO & 1 & 1 & 0 & & 1 & 1 & 1 & \\
\hline Consolidation & 0 & 0 & 0 & & 1 & 0 & 1 & \\
\hline Lobes affected & 5 & 2 & 0 & & 5 & 5 & 5 & \\
\hline \multicolumn{9}{|l|}{ Fibrosis } \\
\hline 1 & 1 & 0 & & 1 & 0 & 1 & & \\
\hline Total Scores & 8 & 4 & 1 & & 11 & 8 & 11 & 0.075 \\
\hline
\end{tabular}

Note: GGO, Ground-Glass Opacities; Negative=0 score; Positive =1 score.

\section{Chest CT Findings}

In the cured group, the lung lesions in all patients showed a significant decrease in absorption, however,2 patients $(2 / 3)$ were still with obvious ground glass or fiber strips (Figure1), but no consolidation were observed. 2 patients in isolation treatment group, multiple GGO, fibrosis and consolidation were observed (Figure 2). Our results showed that chest CT findings GGO and fibrosis had overlap in cured group and isolation group (Figure 2). Two cases in isolation group, the main CT findings were highly similar to that of cured patients.

\section{Scores of CT and Clinical Features}

Table 1 showed that 3 patients in cured group, total scores was 8,4 and 1 respectively. Whereas, in isolation group, total scores of 3 patients was 11,8 and 11 . There are no significant different between these two groups $(\mathrm{p}=0.075)$.

\section{Discussion}

2019 Novel coronavirus pneumonia (2019-NCP) is a serious public health hazard epidemic that broke out in December 2019. Early detection, early isolation and early diagnosis are crucial for 
disease prevention and control. However, as reported by Rothe C et al. [5], asymptomatic virus carriers may also transmit novel coronavirus, which poses a great challenge for disease prevention and control. The latest guidelines in China's NCP (the 5th edition) clearly emphasized asymptomatic can also be a source of infection, and pointed out that suspected cases in Hubei province, CT examination with the same value as nucleic acid detection. But for the case of outside Hubei, still with virus nucleic acid testing for the diagnosis standard. Chinese guidelines pointed out that the NCP lifted isolation and discharge standards as followed:

a) Body temperature returned to normal more than 3 days.

b) Respiratory symptoms improved significantly.

c) Lung imaging showed obvious inflammation absorption.

d) Respiratory pathogen nucleic acid detection on two consecutive negative (sampling time interval of at least 24 hours).

It means that 2019-nCov infected patients would be free according to the guidelines. Meanwhile, because of lack of followup standardized management and follow-up strategy, whether these so-called cure patients have the potential for infection is not yet completely removed. In this study, 2 patients in the cured group was similar to all patients in isolated group and they still had dry cough upon discharge. Although body temperature of these patients returned to normal for 3 days, the symptoms of dry cough still did not disappear. As we known, droplet transmission is the main method for NCP, and the discovery of aerosol transmission is even more worrying. Whether the cough process can lead to the spread of the virus remains to be closely watched. Our results showed that 3 patients in isolation group showed multiple GGO and consolidation in bilateral lung. 2 patients among them showed a small amount of fibrosis with disease progression. These CT findings were consistent with literature reports [6,7]. GGO and consolidation were the main chest CT features in NCP patients, and this feature displayed that there is active inflammation and exudation.

Pulmonary lesions will be obviously or fully absorbed after effective treatment [8]. 3 patients in cured group showed that consolidation was disappear. However, 2 patients were discharged with residual some GGO and fiber strip lesions in lungs, showing overlaps and similarities with GGO changes in the isolation group. This means that the inflammatory exudate is still present in these patients. Further observation should be needed to confirm whether there is still virus residue and whether it can still become the source of infection through coughing after the discharged isolated patients. Our results showed that all these patients in two group, twice coronavirus nucleic acid test were negative. However, 2 patients in cured group and 3 patients in isolation group all displayed bilateral pulmonary lesions. Beyond our expectation, the coronavirus nucleic acid test of 2 patients was positive in isolation group before admission to our hospital as NCP patients. However, after admission, multiple, multi-site (sputum, blood, anus, pharynx) new coronavirus nucleic acid tests were negative, but with more bilateral pulmonary lesions. Many studies also have reported that nucleic acid was negative in many clinically diagnosed patients. We suspect that this result is related to numbers of factors affecting nucleic acid test. Firstly, virus activity and quantity, and the influence of the patient's own immunity, also may be related to the progress of the disease. Secondly, samples from different parts, qualified rate of samples, different reagents and different detection methods have a certain influence on nucleic acid detection results $[9,10]$. some study revealed that the detection positive rate of lower respiratory secretions is higher than that of upper respiratory secretions. In addition, at present, nucleic acid detection is still as gold standard to confirmed NCP, but the sensitivity and specificity of NCP are still not evaluated [9].

According to current clinical observation, the positive rate is only $30 \%-50 \%$. Therefore, two times negative nucleic acid tests for discharged patients (more than 24 hours apart) cannot indicate that the virus has been completely eliminated in patients. In addition, for suspected cases, patients with two negative coronavirus nucleic acid tests may also be infected with the new coronavirus, who cannot be completely isolated. William Kyle Silverstein et al. [11] reported a patient in Canada who was discharged from hospital for two weeks was still detectable with the 2019-nCoV, and moreover, one case reported earlier by Camilla Rothe et al. [5] about transmission of 2019-nCoV infection from an asymptomatic contact in Germany. These cases all indicated that the virus was still present in patients who met with the release and discharge criteria. Therefore, as a matter of fact, these two cases confirms our concern about the scientific nature of the criteria for the release isolation and discharge of the latest clinical practice guidelines of 2019-nCov in China [12].

\section{Limitation and Conclusion}

The main limitations of this study, firstly, the small number of NCP cases enrolled in each group. Besides, Clinical and CT data for patients in cured group follow-up are still lacking. However, based on our results, previous reports and current clinical experience, there are large overlap of main clinical manifestations and CT findings in cured patients and isolated patients. In view of the high infectivity of NCP, the characteristics of the diversification of transmission routes and low sensitivity of nucleic acid, it is extremely worry that NCP patients met with the criteria for the release isolation and discharge of according to the latest clinical practice guidelines of 2019-nCov in China. In our opinion, these socalled cured patients still need to be centralized and standardized in isolation and followed up closely. Even if they are asymptomatic, the new coronavirus nucleic acid test and chest CT examination should be re-examined after 14 days. 


\section{Conflict of Interest}

There is no conflict.

\section{Funding Source}

Science innovative project of Foshan

(FSOAA-KJ218-1301-0021)

\section{Ethical Approval}

Our institutional review board waived written consent for this retrospective case series involved no potential risk to patients.

\section{References}

1. Chen N, Chen N, Zhou M, Dong X, Qu J, et al. (2020) Epidemiological and clinical characteristics of 99 cases of 2019 novel coronavirus pneumonia in Wuhan, China: A descriptive study. The Lancet 395(10223): 507-513.

2. Wang C, Horby PW, Hayden FG, Gao GF (2020) A novel coronavirus outbreak of global health concern. Lancet 395(10223): 470-473.

3. Chan JF, Yuan S, Kok KH, To KK, Chu H, et al. (2020) A familial cluster of pneumonia associated with the 2019 novel coronavirus indicating person-to-person transmission: a study of a family cluster. Lancet 395(10223): 514-523.

\section{ISSN: 2574-1241}

DOI: $10.26717 /$ BJSTR.2020.26.004336

ZF Xu, AZ Pan. Biomed J Sci \& Tech Res

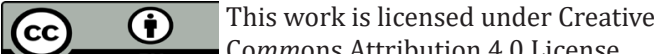

Submission Link: https://biomedres.us/submit-manuscript.php
4. Lin L, TS Li (2020) Interpretation of "Guidelines for the Diagnosis and Treatment of Novel Coronavirus (2019-nCoV) Infection by the National Health Commission (Trial Version 5)". Zhonghua Yi Xue Za Zhi 100(0): E001

5. Rothe C, Schunk M, Sothmann P, Bretzel G, Froeschl G, et al. (2020) Transmission of 2019-nCoV Infection from an Asymptomatic Contact in Germany. N Engl J Med.

6. Pan Y, Guan H (2020) Imaging changes in patients with 2019-nCov. Eur Radiol.

7. Chung M, Bernheim A, Mei X, Zhang N, Huang M, et al. (2020) CT Imaging Features of 2019 Novel Coronavirus (2019-nCoV). Radiology.

8. Shi H, Han X, Zheng C (2020) Evolution of CT Manifestations in a Patient Recovered from 2019 Novel Coronavirus (2019-nCoV) Pneumonia in Wuhan, China. Radiology

9. Xiao SY, Wu Y, Liu H (2020) Evolving status of the 2019 novel coronavirus Infection: proposal of conventional serologic assays for disease diagnosis and infection monitoring Commentary/Review. J Med Virol

10. Is test frequent false negative, which can misdiagnose a large number of new crown pneumonic patient.

11. William Kyle Silverstein, Lynfa Stroud, Graham Edward (2020) First imported case of 2019 novel coronavirus in Canada, presenting as mild pneumonia. Lancet 395(10225): 724.

12. Lei J, Li J, Xiaolong Q (2020) CT Imaging of the 2019 Novel Coronavirus (2019-nCoV) Pneumonia. Radiology.

$\begin{array}{ll}\text { BIOMEDICAL } & \text { Assets of Publishing with us } \\ \text { RESEARCHES } & \text { - Global archiving of articles } \\ \text { - Immediate, unrestricted online access }\end{array}$

